\title{
Quasi-absence de réinfestation par les strongles du bétail gambien en saison sèche
}

\author{
P. Ankers ${ }^{1}$, J. Zinsstag ${ }^{1}$, K. Pfister ${ }^{2}$,
}

ANKERS (P.), ZINSSTAG (J.), PFISTER (K.). Quasi-absence de réinfestation par les strongles du bétail gambien en saison sèche. Revue Élev. Méd. vét. Pays trop., 1994, 47 (2) : 201-205

Cinq taureaux Ndama âgés de 1,5 à 2 ans et 20 béliers Djallonké âgés de 6 à 18 mois, maintenus sur des pâturages naturellement infestés, ont été vermifugés en novembre (début de saison sèche) pour les bovins et en novembre, janvier, mars et mai pour les ovins divisés en 4 groupes. L'excrétion d'eufs de strongles gastro-intestinaux a été déterminée hebdomadairement à partir du traitement. L'excrétion d'eufs est restée pratiquement nulle pour tous les animaux à l'exception de la série d'ovins traités en mai et qui a recommencé à excréter les aufs de strongles gastro-intestinaux un mois après la première pluie. Un examen parasitologique complet, après abattage en juin pour les bovins et dès la neuvième semaine après le traitement pour les ovins, a permis de confirmer l'absence de réinfestation, pathologiquement conséquente, par des strongles gastro-intestinaux du bétail gambien pendant la saison sèche. Ceci confirme l'importance stratégique de l'application d'un vermifuge actif contre les strongles immatures en tout début de saison sèche, et rend superflue toute vermifugation supplémentaire entre novembre et juin.

Mots clés : Bovin - Ovin - Bélier Djallonké - Taureau Ndama - Infestation - Trychostrongylidae - Helminthose gastrointestinale - Anthelminthique - Pâturage - Gambie.

\section{INTRODUCTION}

Les helminthoses sont responsables chez le bétail gambien de pertes économiques considérables qui viennent s'ajouter à celles provoquées par la malnutrition, la trypanosomose et les autres maladies épizootiques. En effet, 97 p. 100 des ruminants sont infestés de strongles gastrointestinaux $(7,12)$ qui causent dans cette zone une perte de poids de $25 \mathrm{~kg}$ chez les bovins à 3 ans (Zinsstag, communication personnelle) et une baisse du gain de poids annuel de 20 à 23 p. 100 chez les moutons à 12 mois (6). Les bovins développent progressivement une immunité à l'infestation par les strongles gastro-intestinaux durant les trois premières années. Les ovins, en revanche, ne développent pas d'immunité mesurable et sont donc porteurs de charges considérables à tout âge (7).

Des études épidémiologiques effectuées en Gambie ont montré que chez les bovins, Haemonchus sp. et Cooperia spp. sont les nématodes les plus rencontrés (12) tandis que chez les ovins, ce sont Trichostrongylus colubriformis, Haemonchus $\mathrm{sp}$. et Oesophagostomum

1. Swiss Helminthosis Project, Centre de trypanotolérance international, PMB 14, Banjul, Gambie.

2. Labor Pfister, Postfach, 3018 Berne, Suisse.

Reçu le 17.8.1993, accepté le 3.5.1994. columbianum (7). La population d'Haemonchus sp. adulte est la plus importante en août-septembre, c'est-à-dire lorsque les précipitations sont maximales. Pendant la saison sèche, les populations d'Haemonchus sp. survivent sous forme de larve hypobiotique chez les bovins et également sous forme adulte chez le mouton.

Les populations de Trichostrongylus spp. et de Cooperia spp. adultes sont, elles aussi, maximales en saison des pluies mais restent toutefois importantes durant la saison sèche avec un nombre d'œufs réduit dans l'utérus des femelles (échelle : 2 000-5 000 parasites adultes par ovin) de décembre à mai $(7,12)$. En Gambie, les charges de Oesophagostomum spp. adultes n'ont pas montré de variations saisonnières.

En conséquence, l'excrétion d'œufs de parasites par gramme de matières fécales (OPG) diminue fortement en fin de saison des pluies, reste basse durant l'essentiel de la saison sèche, puis augmente 'à nouveau 1 à 2 mois avant le début de la saison des pluies suivante, due à une réactivation des larves inhibées ou des adultes en phase hypométabolique (12), appelée "rain rise".

Lors d'une expérience préliminaire, on a obtenu, après application d'un vermifuge actif sur les strongles immatures en décembre sur un demi-troupeau, des OPG inférieurs à ceux des animaux non traités durant tout le reste de la saison sèche, et un report de 2 mois de l'augmentation de l'OPG à la venue des pluies (13). Ce report a atteint 150 j lors d'une autre expérience où la totalité d'un troupeau isolé a été vermifugée en février (Zinsstag, communication personnelle). On peut en déduire qu'il n'y aurait pas de réinfestation par des nématodes gastrointestinaux du bétail gambien en saison sèche. C'est cette déduction que l'expérience décrite dans cet article tente de vérifier afin d'ajuster toute proposition de calendrier de vermifugation.

\section{MATÉRIEL ET MÉTHODE}

\section{Climatologie}

La Gambie, en zone soudano-guinéenne, jouit d'un climat tropical avec une saison des pluies qui s'étend de juin à octobre (fig. 1). Dans la division de "McCarthy Island" où a été conduite l'expérience, la moyenne des précipitations varie entre 650 et $1200 \mathrm{~mm}$ (station ITC, Bansang). 


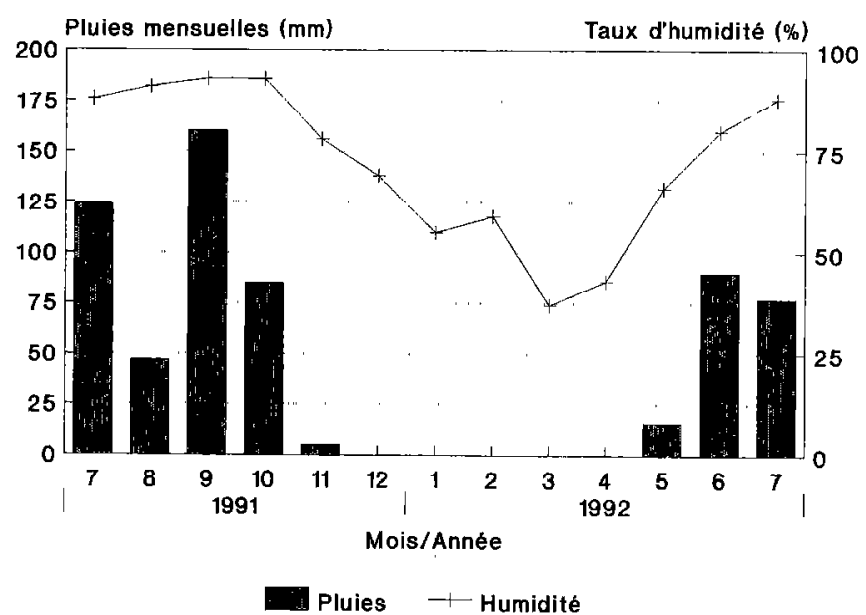

Figure 1 : Pluviométrie et hygrométrie dans la division de McCarthy Island en Gambie (juillet 1991-juillet 1992).

\section{Les animaux de l'expérience}

Cinq taureaux Ndama âgés de 18 à 24 mois, soumis à une infestation naturelle, ont été vaccinés contre le charbon bactéridien, le charbon symptomatique et la pasteurellose. Les animaux ont été vermifugés à l'ivermectine (Ivomec ${ }^{\circledR}, M S D-A G V E T$ ) avec $0,2 \mathrm{mg} / \mathrm{kg}$ par injection sous-cutanée deux fois, à une semaine d'intervalle, au début de la saison sèche (novembre 1991). Ils ont été placés ensuite dans 5 troupeaux villageois en conditions d'élevage traditionnel (pâture libre le jour et retour à un même point d'attache pour la nuit). La répétition du traitement à la dose conseillée par le fabricant a pour but d'assurer l'élimination totale des strongles gastro-intestinaux présents.

Par ailleurs, 20 béliers Djallonké âgés de 6 à 18 mois ont été achetés, vaccinés contre la peste des petits ruminants, puis placés par groupe de 4 dans 5 troupeaux villageois (pâture libre le jour et en enclos la nuit). En novembre, un mouton de chaque groupe a été vermifugé suivant le même schéma que les bovins, constituant ainsi une série de 5 individus vermifugés en même temps mais se trouvant dans 5 troupeaux différents. Les trois autres séries de 5 moutons ont été traitées de la même manière en janvier, mars et mai, respectivement.

Les 5 bovins ont été sacrifiés début juin 1992 (fin de saison sèche) tandis que les ovins ont été sacrifiés par série de 5 dès la 9e semaine après la vermifugation, à un intervalle de 2 mois. L'abattage des ovins, réparti sur toute la saison sèche, permettait de déterminer une éventuelle réinfestation et le moment où celle-ci intervenait. Dès le premier traitement et jusqu'à l'abattage, on a prélevé hebdomadairement, sur chacun des animaux, un échantillon de fèces pour coproscopie qualitative (méthodes de flottation et de sédimentation) (21) et quantitative (méthode de McMaster) (8) pour détecter une éventuelle réapparition des œufs, ainsi qu'un échantillon de sang (bimensuel) afin de déterminer l'hématocrite et détecter une éventuelle trypanosomose par la méthode dite du "fond noir" (18). Des échantillons de fèces d'ovins ( $n=21$ à $45 /$ mois) et de bovins ( $n=14$ à $56 /$ mois), tous du même âge que les animaux de l'expérience et exposés à une infestation naturelle, ont été examinés pour établir une moyenne d'excrétion d'animaux témoins non traités.

\section{Autopsies}

Après abattage, le tractus digestif est lavé, les parasites gastro-intestinaux récupérés et les muqueuses de l'abomasum, du cæcum et $45 \mathrm{~cm}$ du colon, digérés selon la méthode décrite par GRABER et PERROTIN (9).

\section{RÉSULTATS}

\section{Coproscopie}

Chez les bovins de l'expérience, dès la première semaine après traitement, et jusqu'au moment de l'abattage, aucune excrétion d'œufs de strongles gastro-intestinaux n'a été décelée par la méthode de McMaster (fig. 2). En revanche; il a été décelé par flottation une excrétion d'œufs dès mi-mai chez un des animaux. En ce qui concerne les ovins, seuls les cinq béliers vermifugés en mai ont recommencé à excréter des œufs fin juin (fig. 3). Des œufs de Strongyloides papillosus ont été décelés à la flottation, en très faible quantité, chez un mouton de chacune des 3 premières séries, et aussi dès fin juin avec la méthode de McMaster chez tous les animaux de la série abattue mi-juillet. La moyenne des OPG des animaux témoins reste basse durant touto la saison sèche puis s'élève progressivement quelques semaines avant la première pluie ("rain rise").

Parallèlement à la recherche d'œufs de nématodes, on a compté les oocystes de coccidies. Les taux d'oocystes de coccidies le jour du traitement, une semaine, puis 2 et 6 semaines après traitement, ont été comparés afin de déterminer si une vermifugation influençait le développement des coccidies. Chez les 5 bovins et chez 19 moutons sur 20, aucune augmentation significative du taux d'oocystes n'a pu être constatée. L'excrétion d'oocystes d'un mouton a augmenté de façon significative une semaine après traitement pour redevenir normale la deuxième semaine.

\section{Autopsies}

On a trouvé, au total, chez les 5 bovins sacrifiés en juin, 6 Haemonchus placei (stade L4, juvéniles et adultes confondus), 6 Cooperia sp., 2 Oesophagostomum radiatum et 1 Trichuris globulosa). On n'a trouvé ni larve d'Haemonchus sp. dans la muqueuse abomasale, ni larve $\mathrm{L} 4$ d'Oesophagostomum radiatum dans la muqueuse cæcale. Pour les 15 ovins sacrifiés durant la saison 


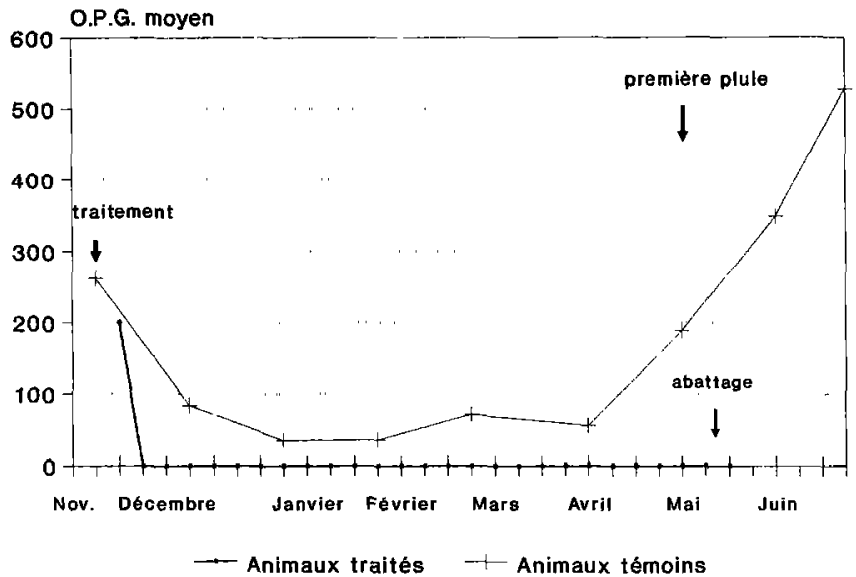

Figure 2 : Elimination des ceufs de strongles (OPG) dans les matières fécales des ovins vermifugés et témoins en saison sèche (Méthode de McMaster).

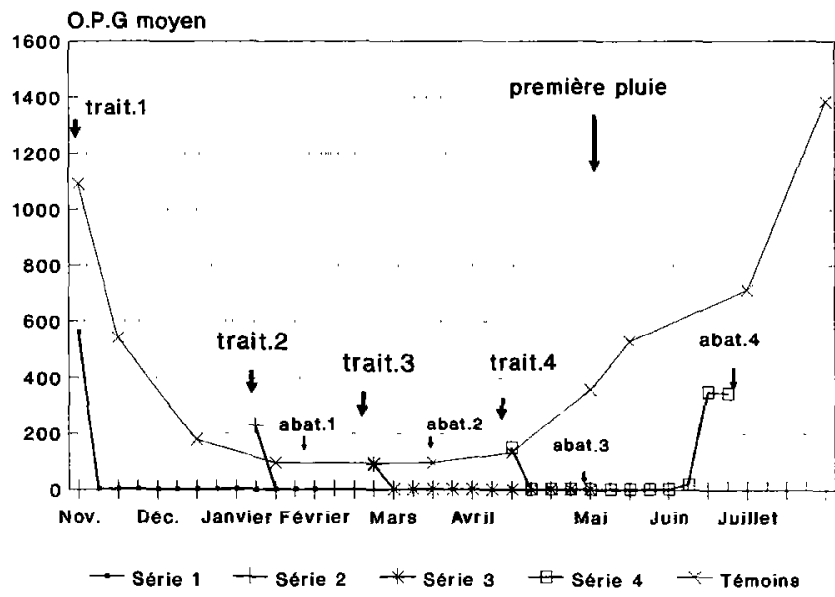

Figure 3 : Elimination des aufs de strongles (OPG) dans les matières fécales des bovins vermifugés et témoins en saison sèche (Méthode de McMaster).

sèche, on a retrouvé 1 Haemonchus contortus adulte et 1 larve dans la muqueuse abomasale chez 2 moutons sacrifiés en février. Ce n'est que chez les 5 moutons sacrifiés mi-juillet (après le début des pluies) que l'on constate une nette réinfestation par $H$. contortus (étendue : 30-108). D'autre part, 11 des 20 ovins sacrifiés étaient porteurs de Trichuris ovis (étendue : 1-11) et 3 contenaient un nombre faible de Strongyloides papillosus) (étendue : 1-11). Une seule larve L4 d'Oesophagostomum sp. a été trouvée dans la muqueuse du caecum d'un mouton sacrifié en avril. Quatre des cinq bovins et 19 des 20 moutons étaient infestés par des ténias anoplocéphalidés. Douze moutons étaient porteurs de Cysticercus taenuicollis et tous les taurillons étaient porteurs de Paramphistomum sp.

\section{DISCUSSION}

La saisonnalité des nématodes gastro-intestinaux les plus fréquents en Gambie est similaire à ce qui est décrit dans d'autres pays d'Afrique de l'Ouest au-dessus du 11e parallèle et même au centre du Togo, c'est-à-dire une présence, toute l'année, de Cooperia spp. adultes avec tout de même une baisse de la population en saison sèche, comme par exemple au Sénégal (15) et au Togo (4), et le maintien d'une population de Trichostrongylus spp. adultes pendant toute l'année, ainsi que la survie d'Haemonchus sp. essentiellement sous forme hypobiotique mais parfois sous forme adulte comme au Sénégal $(15,22)$, au Niger $(20)$, au Nord-Nigeria $(10,16)$ et au Togo (4). Pour ces 3 genres, les femelles excrètent des œufs pendant toute l'année, même si c'est en nombre réduit (12). En dessous du 11e parallèle, leur saisonnalité est moins marquée ou carrément inversée comme en Sierra Leone (1) et on observe une réinfestation pendant toute l'année, comme au sud du Nigeria $(5,17)$, au Ghana (2) et au Bénin (14).

En ce qui concerne Oesophagostomum spp., une variation saisonnière des charges, telle qu'elle est décrite par exemple au Sénégal (15), au Niger chez le mouton (20) et même au Togo (4), n'a pas été observée en Gambie chez les bovins et les ovins $(7,12)$.

Peut-on considérer que cette saisonnalité est liée à l'absencc de réinfestation? Dans le cas de cette expérience, les 8 Haemonchus sp., les 3 Oesophagostomum sp. (L4, juvénile et adulte) et les 6 Cooperia sp., mais aussi les 12 Strongyloides papillosus trouvés sur l'ensemble des 5 taurillons et 15 moutons abattus avant les pluies, ne permettent pas d'affirmer qu'il n'y a pas du tout de réinfestation du bétail en saison sèche mais démontrent que s'il y a réinfestation, celle-ci est négligeable.

L'OPG des animaux témoins quadruple déjà dès le mois de mai (rain rise) ce qui, si le milieu extérieur permet l'accomplissement de la phase exogène des cycles parasitaires, va engendrer la réinfestation du bétail. On n'a toutefois pas retrouvé de larves infestantes dans l'herbe des points d'attache nocturne avant juillet 1992. Les taurillons ont pu se réinfester lors de l'abreuvement en des points d'eau de pluies résiduelles puisqu'une première pluie est tombée 17 jours avant leur abattage (11). La possibilité que ces quelques parasites aient survécu à la double vermifugation n'est pas à exclure.

Seuls les ovins abattus en juillet, c'est-à-dire un mois et demi après la première pluie, sont clairement porteurs, de strongles gastro-intestinaux suite à une réinfestation. Toutefois, la charge parasitaire de ces animaux est encore faible à ce moment-là et n'atteint pas le seuil favorisant le développement de symptômes pathologiques. La présence régulière (prévalence de $55 \mathrm{p}$. 100) d'un petit nombre de Trichuris ovis chez les ovins s'explique par la bonne capacité de survie des œufs à la dessiccation et par l'efficacité limitée de l'ivermectine contre le stade 
adulte de cette espèce (évaluée à 87 p. 100 ) (3). Cette prévalence, beaucoup plus élevée que celle trouvée par FRITSCHE (4 p. 100) (7) sur des animaux non traités, peut s'expliquer par la technique de post mortem utilisée. La lecture de la totalité du contenu du cæcum et du colon ascendant au lieu d'une partie aliquote, permet de déceler toute présence de vers, si limitée soit-elle. La présente expérience ne permet pas d'évaluer les risques de réinfestation par Gaigeria pachyscelis chez le mouton, étant donné leur longue prépatence.

\section{CONCIUSION}

Ces résultats montrent la quasi-absence de réinfestation du bétail gambien en saison sèche par les strongles gastro-intestinaux les plus importants. Dans des zones où, comme dans la région de l'étude, les nématodes sont les principaux responsables des parasitoses gastro-intestinales, cette expérience justifie au niveau purement épidémiologique, l'application d'un vermifuge actif sur les strongles immatures en tout début de saison sèche qui va libérer le bétail de ses nématodes jusqu'à la saison des pluies suivantes. Ce traitement pourrait être ajouté à un double traitement annuel à 2 mois d'intervalle pendant les pluies, comme préconisé et déjà utilisé en Gambie (23). Mais l'opportunité économique et le degré d'acceptabilité par les éleveurs de ce troisième traitement de début de saison sèche restent à évaluer.

\section{REMERCIEMENTS}

Cette étude a été réalisée grâce à une bourse allouée par la Coopération au développement et aide humanitaire du gouvernement suisse (DDA). Nous remercions le Centre international de trypanotolérance pour les facilités accordées lors de cette réalisation. Nous remercions les techniciens du projet helminthoses de l'Université de Neuchâtel à Bansang, les éleveurs et gardiens de troupeaux de la région de Bansang pour leur aide et leur coopération ainsi que M. Hans Wagner (ITC) pour la mise à disposition des données concernant les ovins non vermifugés.

\section{BIBLIOGRAPHIE}

1. ASANJ (M.F.), WILLIAMS (M.O.). A qualitative and quantitative survey and seasonal dynamics of gastrointestinal helminth parasites of livestock in Sierra Leone. Bull. Anim. Hlth Prod. Afr., 1987, 35: 191-199.

2. ASSOKU (R.K.G.). Gastrointestinal helminth parasites of cattle in Ghana. Bull. Anim. Hlth Prod. Afr., 1983, 31: 223-230.

3. BENZ (G.W.), RONCALLI (R.A.), GROSS (S.J.). Use of ivermectin in cattle, sheep, goats and swine. In: Ivermectin and Abamectin. William C. Campbell ed., New York, Spinger Verlag, 1989. p. 215-229.

4. BONFOH (B.). Epidémiologie des nématodes gastro-intestinaux chez les petits ruminants de race Djallonké au Togo (région des plateaux). Thèse doct. Dakar, EISMV, 1993. 120 p.
5. FAKAE (B.B.). Seasonal changes and hypobiosis in Haemonchus contortus infection in the West african Dwarf sheep and goats in the nigerian derived savanna. Vet. Parasitol., 1990, 36 (1-2): 123-130.

6. FAUGERE (O.), TILLARD (E.), FAUGERE (B.). Prophylaxie chez les petits ruminants au Sénégal : évaluation technico-économique de leurs effets en milieu villageois. Dakar, Sénégal, LNERV, 1991. 212 p. (document de travail PPR $\mathrm{n}^{\circ} 34$ )

7. FRITSCHE (T.), KAUFMANN (J.), ZINSSTAG (J.), PFISTER (K.). The seasonal epidemiology of gastrointestinal parasites of small ruminants in the Gambia. Vet. Parasitol, 1993, 49: 271-283.

8. GORDON (H.Mcl.), WHITLOCK (H.V.). A new technique for counting nematode eggs in sheep faeces. J. Counc. scient. ind. Res. Aust., 1939, 12: $50-52$.

9. GRABER (M.), PERROTIN (C.). Helminthes et helminthoses des ruminants domestiques d'Afrique tropicale. Point vét., 1983.378 p.

10. HART (J.A.). Observations on the dry season strongyle infestations of zebu cattle in Northern Nigeria. Br. vet. J., 1964, 120: 87.

11. KAUFMANN (J.), PFISTER (K.). In: Second progress report. The Gambia, ITC-University of Bern helminthosis Project, 1990. p. 22.

12. KAUFMANN (J.), PFISTER (K.). The seasonal epidemiology of gastrointestinal nematodes in Ndama cattle in the Gambia. Vet. Parasitol. 1990, 37: 45-54.

13. KAUFMANN (J.), PFISTER (K.). In: Third progress report. The Gambia, ITC-University of Bern helminthosis Project, 1990. p. 11.

14. LADIKPO (E.). Nématodoses digestives des veaux en République populaire du Bénin. Paris, Agence de Coopération culturelle et technique, 1984. $158 \mathrm{p}$.

15. N'DAO (M.). Contribution à l'étude de l'épidémiologie des nématodes gastro-intestinaux des ruminants dans la zone sylvo-pastorale du Sénégal. Thèse doct. Dakar, EISMV, 1991, 125 p.

16. OGUNSUSI (R.A.), EYSKER (M.). Inhibited development of trichostrongylids of sheep in northern Nigeria. Res. vet. Sci, 1979, 26: 108-110.

17. OKON (E.D.), AKINEPLU (A.I.). Development and survival of nematode larvae on pasture in Calabar, Nigeria. Trop. Anim. Hlth. Prod., 1982, 14: $23-25$.

18. PARIS (J.), MURRAY (M.), McODIMBA (F.). A comparative evaluation of the parasitological techniques currently available for diagnosis of African trypanosomiasis in cattle. Acta trop, 1982, 39: 307-316.

19. SCHILLHORN VAN VEEN (T.W.). Haemonchosis in sheep during the dry season in the Nigerian savanna. Vet. Rec., 1978, 102: 364-365.

20. TAGER-KAGAN (P.). Les principales helminthiases des animaux domestiques au Niger et leur traitement. Niger, INRA, 1982. 33 p.

21. URQUHART (G.M.), ARMOUR (J.), DUNCAN (J.L.), DUNN (A.M.) JENNINGS (K.W.). Veterinary parasitology. New York, Churchill Livingstone Inc. 1987.

22. VERCRUYSSE (J.). The seasonal prevalence of inhibited development of Haemonchus contortus in sheep in Senegal. Vet. Parasitol., 1985, 17: 159-163.

23. ZINSSTAG' (J.), KAUFMANN (H.), PFISTER (K.). Improved productivity of gambian N'dama cattle through strategic control of helminthosis. In: Proc of the 7th international conference of institutions of tropical veterinary medicine, Yamoussoukro, sept. 1992, Vol. II. Feldafing, DSE, Maisons-Alfort, CIRAD-EMVT, 1993. p. 575-581. 
ANKERS (P.), ZINSSTAG (J.), PFISTER (K.). Quasi-absence of trichostrongylid reinfection in Gambian livestock during the dry season. Revue Élev. Méd. vét. Pays trop., 1994, 47 (2) : 201-205

Five Ndama bulls aged between 1.5 and 2 years and 20 Djallonke rams aged between 6 to 18 months were dewormed as follows: the bulls were dewormed in November (beginning of the dry season), the sheep were divided into four groups dewormed in November, January, March and May respectively. The animals were kept on naturally infected pastures prior to and throughout the experiment. Gastrointestinal nematode egg excretion was measured weekly after starting the treatment. Egg excretion stayed practically nil for all animals except for the series of sheep treated in May which started excreting eggs on month after the first rain. The bulls were slaughtered in June and the sheep were slaughtered nine weeks after they had been initially dewormed. A complete parasitological post mortem examination confirmed the absence of any pathologically consequent reinfection by gastro-intestinal nematodes of Gambian cattle during the dry season. This confirms the strategic importance of the application of a dewormer also efficient against immature trichostrongylids at the beginning of the dry season and renders complementary deworming between November and May superfluous.

Key words : Cattle - Sheep - Djallonké ram - Ndama bull - Infestation Trichostrongylid - Gastro-intestinal helminthosis - Anthelmintics Pasture -The Gambia.
ANKERS (P), ZINSSTAG (J.), PFIS'IHK (K.). Ausencia casi total de re-infestación por estróngilos en el ganado gambiano durante la estación seca. Revue Élev. Méd. vét. Pays trop., 1994, 47 (2) : 201-205

Se desparasitaron cinco toros Ndama de 1,5 a 2 años y veinte carneros Djallonké, de 6 a 18 meses de edad, mantenidos en pastizales infestados naturalmente. Las desparasitaciones en los bovinos se llevaron a cabo en noviembre (principio de la época seca) y los ovinos se dividieron en 4 grupos, desparasitados en noviembre, enero, marzo y mayo. La excreción de los luevos de estróngilos gastruintestinales se determinó semanalmente a partir del tratamiento. La excreción de huevos fue practicamente nula para todos los animales, a excepción del grupo de ovinos tratados en mayo, el cual comenzó de nuevo a excretar huevos de estróngilos gastrointestinales un mes después de la primera lluvia. En matadero, el examen parasitológico completo llevado a cabo en junio para los bovinos, y a partir de nueve semanas post-tratamiento para los ovinos, confirmó la ausencia de re-infestaciones patológicamente significativas por estróngilos gastrointestinales en el ganado gambiano durante la estación seca. Esto confirma la importancia estratégica de la administración del antihelmíntico dirigido contra los estróngilos inmaduros a principios de la estación seca, restando importancia a cualquier otra desparasitación suplementaria, efectuada entre noviembre y junio.

Palabras clave : Bovino - Ovino - Morueco Djalonke - Toro Ndama Infestación - Trichoestrongilo - Gastrointestinal helmintosis - Antihelmíntico - Pasto - Gambia 